\title{
Inclusive Search for Standard Model Higgs Boson Production in the WW Decay Channel Using the CDF II Detector
}

\author{
Massimo Casarsa* \\ (on behalf of the CDF Collaboration)
}

INFN, Trieste, Italy

E-mail: massimo.casarsadts.infn.it

\begin{abstract}
The search for the Standard Model Higgs boson at the Tevatron has been highly successful in driving the first Higgs exclusions since LEP. This search by CDF is performed using the full Run II dataset and looks specifically into the Higgs to $W^{+} W^{-}$final state of two charged leptons $(e, \mu)$ and two neutrinos from the collision of proton-antiproton pairs at $\sqrt{s}=1.96 \mathrm{TeV}$. To maximize signal acceptance, events with same-sign dileptons and trileptons are included as separate regions to account for associated Higgs production with a $Z$ or $W$ boson and for Higgs production via vector boson fusion. Additionally, events with low dilepton invariant mass and events with hadronic taus further increase the signal acceptance. The search excludes at the $95 \%$ confidence level a Standard Model Higgs boson in the mass range between $148 \mathrm{GeV} / c^{2}$ and $175 \mathrm{GeV} / c^{2}$ and is also one of the main contributors to the Tevatron's sensitivity to the low mass Standard Model Higgs boson.
\end{abstract}

36th International Conference on High Energy Physics

4-11 July 2012

Melbourne, Australia

\footnotetext{
* Speaker.
} 


\section{Introduction}

The Higgs mechanism [1] was introduced in the Standard Model (SM) of particles physics [2] to account for the electroweak symmetry breaking and the generation of fermion and weak boson masses. As a by-product, it predicts the existence of a scalar boson, the Higgs boson $(H)$, which has been awaiting for an experimental confirmation for almost fifty years. Although the Higgs boson mass cannot be predicted by theory, indications on its value are provided by indirect constraints from the $W$ boson and the top quark masses and by precision global electroweak fits, which seem to prefer a relatively light Higgs [3]: the preferred value for $m_{H}$ is $94{ }_{-24}^{+29} \mathrm{GeV} / c^{2}$ and $m_{H}$ is expected to be lower than $152 \mathrm{GeV} / c^{2}$ at $95 \%$ confidence level (C.L.). The recent discovery of a new boson by the ATLAS and CMS Collaborations at LHC with a mass of approximately 126 $\mathrm{GeV} / c^{2}$ [4 and an excess of events in the range between $120 \mathrm{GeV} / c^{2}$ and $135 \mathrm{GeV} / c^{2}$ found by $\mathrm{CDF}$ and $\mathrm{D} \varnothing$ at the Tevatron [5] could put an end to the long quest.

In proton-antiproton collisions at a center of mass energy of $1.96 \mathrm{TeV}$ the SM Higgs boson is predominantly produced via a QCD gluon-gluon fusion process which proceeds through a virtualquark loop, where the main contribution is given by the massive top quark. The cross section for this process decreases from $1.861 \mathrm{pb}$ to $0.192 \mathrm{pb}$ as the Higgs boson mass varies between 100 $\mathrm{GeV} / \mathrm{c}^{2}$ and $200 \mathrm{GeV} / \mathrm{c}^{2}$ [6]. The rates of the electroweak Higgs boson production mechanisms in association with a $W$ or $Z$ vector boson and via vector boson fusion (VBF) are about an order of magnitude lower [7]. Therefore, the mass range $100-200 \mathrm{GeV} / c^{2}$ represents the most sensitive region for a direct search for a SM Higgs boson at the Tevatron.

This contribution reports on the inclusive search by CDF for a SM Higgs boson production in the $W^{+} W^{-}$decay channel, where the $W^{ \pm}$bosons can be virtual, using the full Tevatron Run II dataset, which corresponds to an integrated luminosity of $9.7 \mathrm{fb}^{-1}$. It is organized as follows: Section 2 briefly describes the experimental apparatus, Section 3 outlines the analysis strategy and presents the search results, and in Section 7 the conclusion is drawn.

\section{Experimental apparatus}

The data were recorded with the CDF II detector at the Tevatron collider, a superconducting synchrotron operating at the Fermi National Accelerator Laboratory. The Tevatron accelerated beams of protons and antiprotons to an energy of $980 \mathrm{GeV}$ and collided them at two interaction points, where the CDF and DØ detectors were located. During the Tevatron Run II, between 2002 and $2011,12 \mathrm{fb}^{-1}$ of data were delivered per experiment, approximately $10 \mathrm{fb}^{-1}$ of which were acquired and are available for analysis.

CDF was a multipurpose central detector with a silicon microvertex tracker, a central opencell drift chamber immersed in a superconducting solenoidal magnetic field, electromagnetic and hadronic calorimeters surrounding the tracking system, and muon detectors in the outermost part. A detailed descriptions of the detector can be found in Ref. [8]. Conventionally, the detector geometry is characterized using the azimuthal angle $\varphi$ and the pseudorapidity $\eta=-\ln [\tan (\vartheta / 2)]$, where $\vartheta$ is the polar angle relative to the proton beam axis. The transverse momentum $p_{T}$ and the transverse energy $E_{T}$ are defined as $p \sin \vartheta$ and $E \sin \vartheta$, respectively. 

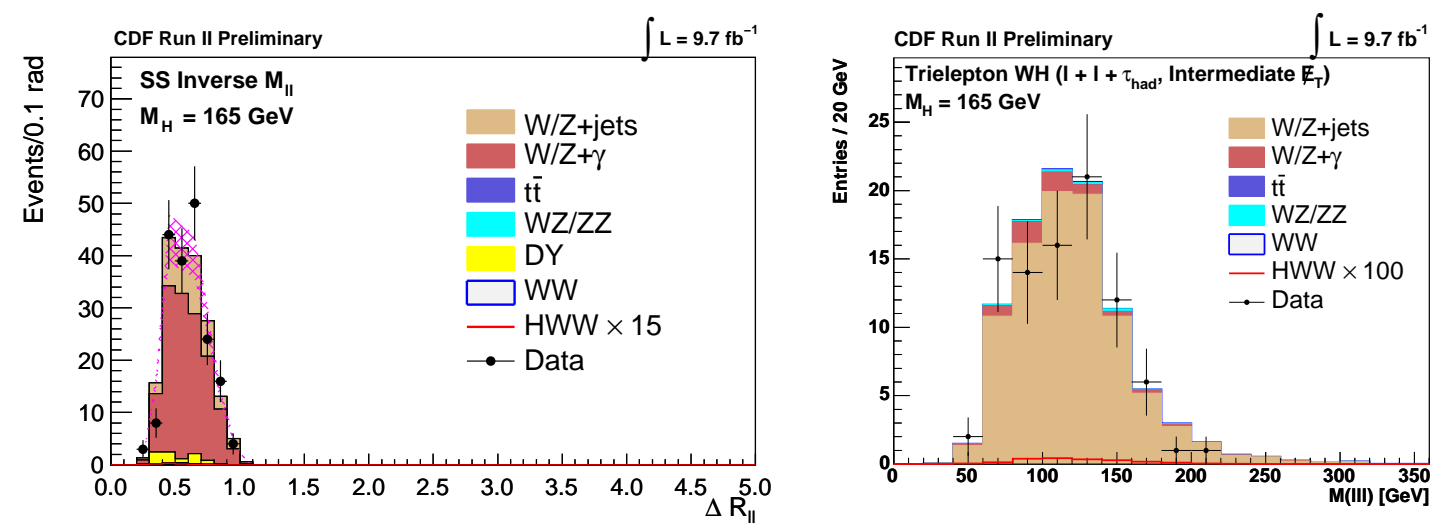

Figure 1: Opening angle $\Delta R_{\ell \ell}$ between the two leptons in the same-sign dilepton channel with $M_{\ell \ell}<16$ $\mathrm{GeV} / \mathrm{c}^{2}$ (left) and invariant mass of the two leptons and the hadronic tau in the trilepton channel with hadronic taus (right). $\Delta R_{\ell \ell}$ is defined as $\sqrt{\Delta \varphi_{\ell \ell}^{2}+\Delta \eta_{\ell \ell}^{2}}$.

\section{Search for a Standard Model Higgs Boson in the WW Decay Channel}

The final state with two $W$ bosons is the dominant decay mode for a SM Higgs boson having a mass greater that $135 \mathrm{GeV} / \mathrm{c}^{2}$ and represents the most sensitive search channel at the Tevatron. The $W^{+} W^{-}$channel is primarily sensitive to the direct gluon-fusion production and provides a clean search topology: leptonic $W$ decays are characterized by high- $p_{T}$ leptons, which provide well-defined experimental signatures. On the other hand, only a partial candidate reconstruction is achievable due to the two neutrinos, which escape detection. For lower SM Higgs boson masses the decay to $b \bar{b}$ dominates, which presents different features and requires a different approach.

The CDF search for the $W^{+} W^{-}$final state is performed inclusively for all the SM Higgs production modes with a significant rate: gluon-gluon fusion, associated production with a $W$ or $Z$ gauge boson, and vector boson fusion, in which a pair of $W$ or $Z$ bosons fuse to form a Higgs boson. The total number of Higgs bosons into $W^{+} W^{-}$expected per delivered $\mathrm{fb}^{-1}$ of data is 49 in the hypothesis $m_{H}=165 \mathrm{GeV} / c^{2}$ and 27 for $m_{H}=125 \mathrm{GeV} / c^{2}$. In the following, the term lepton refers to a reconstructed electron $(e)$ or muon $(\mu)$, whereas with hadronic tau $\left(\tau_{\text {had }}\right)$ we indicate the reconstructed decay of a tau into hadrons and a tau neutrino. The search strategy is mainly driven by the expected small signal-background ratio and consists in a loose kinematical event selection, followed by multivariate analysis (MVA) techniques for a more effective signal-background separation rather than a simple event counting. In order to further enhance the sensitivity, the analysis is conducted on multiple independent data samples, whose selection is targeted to specific production mechanisms, and successively the results are combined. Events with opposite-sign dileptons and zero or one jet, events with opposite-sign dileptons and an inverted requirement on the dilepton mass (i.e. $M_{\ell \ell}<16 \mathrm{GeV} / c^{2}$ ), events with opposite-sign electron-hadronic tau and muon-hadronic tau pairs are most sensitive to the direct production. On the contrary, events with opposite-sign dileptons and two or more jets, events with same-sign dileptons and one or more jets, events with three leptons or two leptons and a hadronic tau are most sensitive to the associated production $W H$ and $\mathrm{ZH}$ and the vector boson fusion.

The data were collected with an online selection which required the presence of a high- $p_{T}$ 

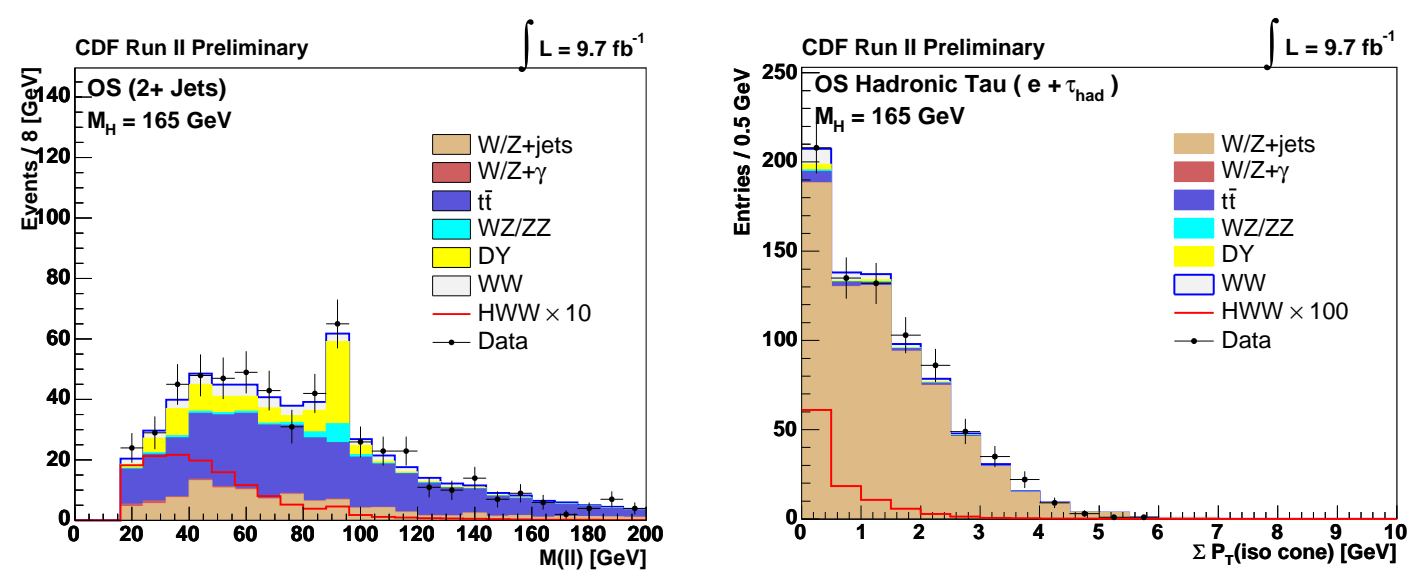

Figure 2: Two-lepton invariant mass in the opposite-sign dilepton channel with two or more jets (left) and scalar sum of $p_{T}$ 's of the tracks inside a 0.3 -radius cone around the tau direction in the $e$ - $\tau_{\text {had }}$ channel (right).

electron or muon (trigger lepton) in the event. Successively, a basic event selection has been applied which requires two or three charged lepton candidates (one of which can be replaced by a hadronic tau), originating from the same vertex, with $p_{T}>20 \mathrm{GeV} / c$ for the trigger lepton and $p_{T}>10$ $\mathrm{GeV} / c$ otherwise $\left(p_{T}>15 \mathrm{GeV} / c\right.$ for the hadronic taus), and a large imbalance in the measured transverse energy within the event, associated with the unobserved neutrinos.

The selected events consist primarily of background SM processes, which are typically on the order of a hundred times larger than the expected signal. In the presence of a small signal over an overwhelming background, advanced MVA techniques, which best exploit the event information, allow an improved signal-background discrimination. The MVA methods rely on an accurate modeling for all processes. In this analysis the $W W$ non-resonant production is modeled with MC@NLO [9], PYTHIA [10], and ALPGEN [11]; WZ and ZZ with PYTHIA and ALPGEN; the Drell-Yan process with PYTHIA, ALPGEN, and MADGRAPH [13]; $t \bar{t}$ with PYTHIA; $W / Z+\gamma$ with the Baur-Berger generator [12] and MADGRAPH; $W / Z+$ jets with data driven techniques and ALPGEN. The Monte Carlo (MC) samples are processed with a GEANT3-based simulation of the CDF II detector response [14] and reconstructed as data, then normalized to the highest-order theoretical cross-section calculation available. Background-enriched samples are used to check and, if necessary, to tune the modeling of the most relevant background processes: they are selected inverting one of the analysis requirements, in order to have a similar selection to the search sample, but with negligible signal content. As an example, Fig. 1 shows the data-MC comparison for the opening angle between the two leptons in the $W / Z+\gamma$ control sample and the invariant mass of the two leptons and the hadronic tau in the $W / Z+$ jets control sample. In the case of the irreducible $W W$ background, which is kinematically very similar to the signal, it is not possible to identify a suitable control region, hence the Monte Carlo modeling for this process is validated by measuring the diboson production cross section in the search sample using the same analysis procedure [15].

The background composition of the selected sample is estimated to be: $1368 \pm 103 \mathrm{WW}$, $243 \pm 14 W Z$ and $Z Z, 424 \pm 46 t \bar{t}, 1135 \pm 168$ Drell-Yan, $2292 \pm 145 W / Z+$ jets, and $498 \pm 35$ $W / Z+\gamma$, for a total of $5961 \pm 252$. For a SM Higgs boson with $m_{H}=165 \mathrm{GeV} / c^{2}$, we expect 

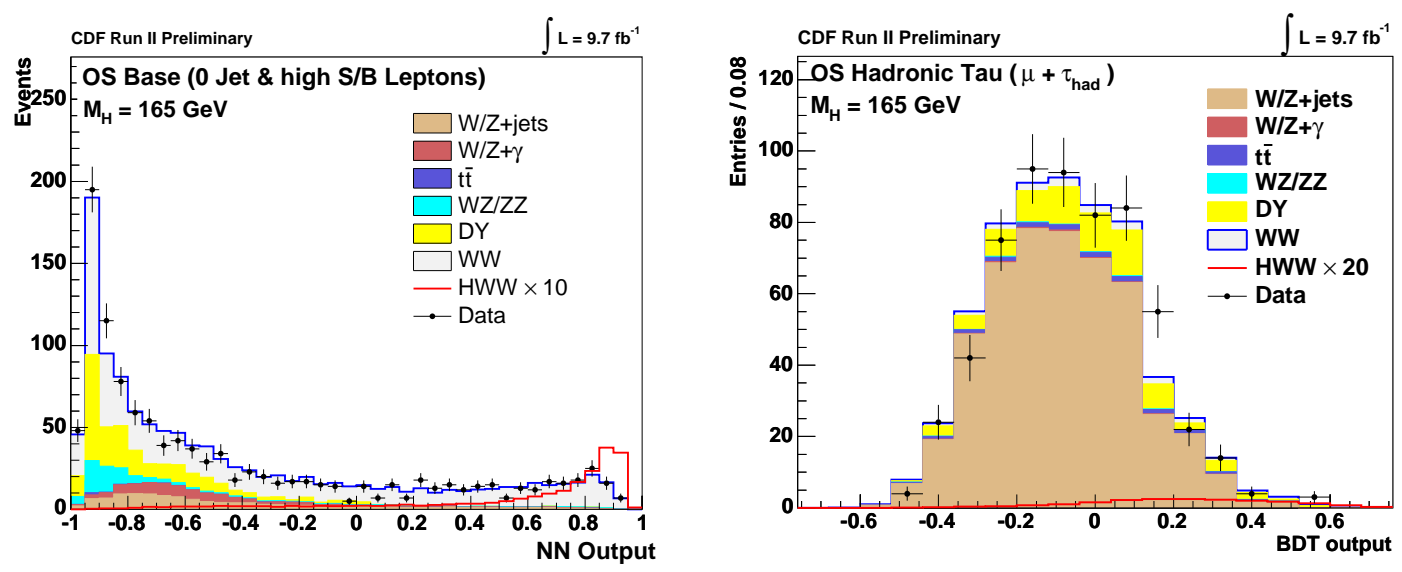

Figure 3: Artificial neural network output for the opposite-sign dilepton channel with no jets (left) and boosted-decision-tree output for the $\mu$ - $\tau_{\text {had }}$ channel (right), in the hypothesis $m_{H}=165 \mathrm{GeV} / c^{2}$.

$54 \pm 11$ gluon-gluon fusion events, $11.6 \pm 0.8 \mathrm{WH}, 5.8 \pm 0.4 \mathrm{ZH}$, and $4.4 \pm 0.5 \mathrm{VBF}$, for a total of $76 \pm 11$ events. The expected number of events for $m_{H}=125 \mathrm{GeV} / c^{2}$ decreases to 18 . In data we observe 6071 events.

The MVA techniques used in this search are the artificial neural network (ANN) [16], the boosted decision tree (BDT) [17], and the matrix element method (ME), which is based on an event-by-event calculation of the probability density for each contributing process to produce the observed event. ANN and BDT utilize kinematical, event-global and particle-identification variables as inputs. Fig. 2 shows the distributions of the most discriminating observable for a Higgs mass of $165 \mathrm{GeV} / c^{2}$ for the opposite-sign dilepton channel with two or more jets and for the $e$ $\tau_{\text {had }}$ channel. The MVA algorithms are optimized and trained to distinguish a $H \rightarrow W^{+} W^{-}$signal from the SM backgrounds for nineteen test masses of the Higgs boson from $110 \mathrm{GeV} / c^{2}$ to 200 $\mathrm{GeV} / \mathrm{c}^{2}$. Two representative distributions of the MVA outputs are shown in Fig. B. the ANN output for the opposite-sign dilepton channel with no jets and the BDT output for the $\mu$ - $\tau_{\text {had }}$ channel. A signal would appear as an excess of events in the data over the expected background in the high score region of the MVA output distributions. Since no significant excess is seen, the MVA distributions are used to set upper limits on the SM Higgs production cross section using a Bayesian method [18]. The combined result excludes a SM Higgs boson with a mass between $148 \mathrm{GeV} / \mathrm{c}^{2}$ and $175 \mathrm{GeV} / \mathrm{c}^{2}$ at $95 \%$ C.L. Fig. $\sharp$ shows the expected and observed upper limits on the SM Higgs boson production cross section as a function of the Higgs boson mass.

\section{Conclusion}

The full CDF Run II dataset, corresponding to $9.7 \mathrm{fb}^{-1}$ of $p \bar{p}$ data, has been analysed in search for a Standard Model Higgs boson decaying to a pair of $W$ bosons exploiting different experimental signatures. The combined analysis achieves sensitivity to the expected SM Higgs production cross section over the mass range $154 \mathrm{GeV} / c^{2}<m_{H}<176 \mathrm{GeV} / c^{2}$. No evidence for a signal is found and upper limits are set on the SM Higgs production cross section, which exclude at 95\% C.L. the mass range $148 \mathrm{GeV} / c^{2}<m_{H}<175 \mathrm{GeV} / c^{2}$. 


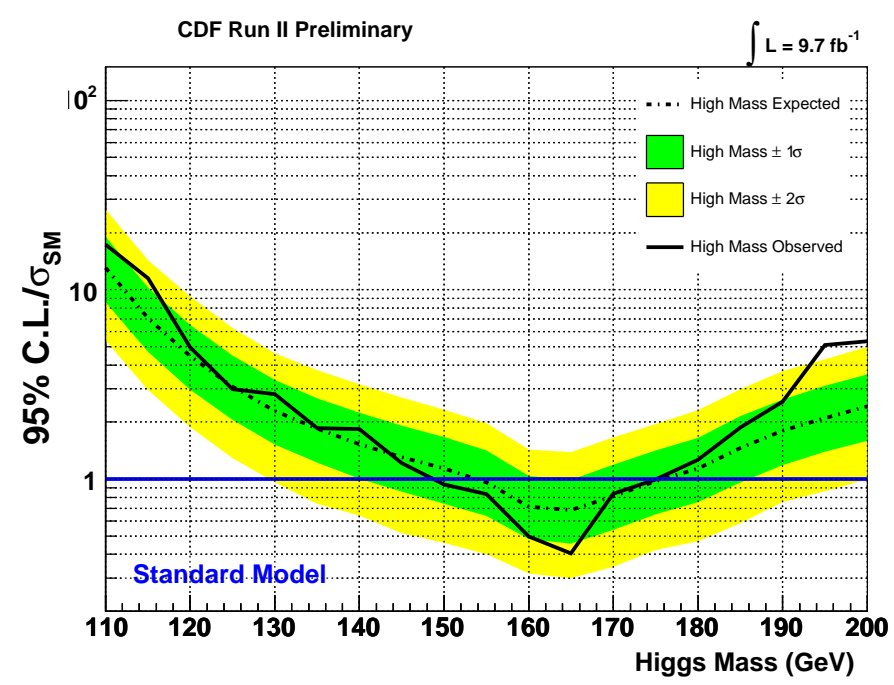

Figure 4: Expected and observed 95\% C.L. upper limits on the SM Higgs boson production cross section as a function of the Higgs boson mass. The limits are expressed as a multiple of the SM prediction for each test mass. The green and yellow bands indicate the $68 \%$ and $95 \%$ probability regions where the limits can fluctuate in the absence of a signal.

\section{References}

[1] F. Englert and R. Brout, Phys. Rev. Lett. 13 (1964) 321; P.W. Higgs, 13 (1964) 508; G.S. Guralnik, C.R. Hagen, and T.W.B. Kibble, 13 (1964) 585; P.W. Higgs, Phys. Rev. 145 (1966) 1156.

[2] S.L. Glashow, Nucl. Phys. 22 (1961) 579; S.Weinberg, Phys. Rev. Lett. 19 (1967) 1264; A. Salam, Elementary Particle Theory, edited by. N. Svartholm (Almqvist and Wiksell, Stockholm 1968), p. 367.

[3] J. Alcaraz et al., http://lepewwg.web.cern.ch/LEPEWWG/.

[4] ATLAS Collaboration, Phys. Lett. B 716 (2012) 1; CMS Collaboration, Phys. Lett. B 716 (2012) 30.

[5] CDF and DØ Collaborations, Phys. Rev. Lett. 109 (2012) 071804.

[6] D. de Florian and M. Grazzini, Phys. Lett. B 674 (2009) 291, arXiv:0901.2427 [hep-ph].

[7] U. Aglietti et al., arXiv:0612172v2 [hep-ph].

[8] CDF Collaboration, Phys. Rev. D 71 (2005) 032001.

[9] S. Frixione and B.R. Webber, J. High Energy Phys. 06 (2002) 029.

[10] T. Sjöstrand, S. Mrenna, P. Skands, J. High Energy Phys. 05 (2006) 026.

[11] M. Mangano, M. Moretti, F. Piccinini, R. Pittau, and A. Polosa, J. High Energy Phys. 07 (2003) 001.

[12] U. Baur, E.L. Berger, Phys. Rev. D 47 (1993) 4889.

[13] J. Alwall et al., J. High Energy Phys. 09 (2007) 028.

[14] R. Brun, R. Hagelberg, M. Hansroul, and J. Lassalle, CERN-DD-78-2-REV (1993).

[15] CDF Collaboration, Phys. Rev. Lett. 104 (2010) 201801.

[16] M. Feindt and U. Kerzel, Nucl. Instrum. Meth. A 559 (2006) 190.

[17] A. Hoecker et al., PoS ACAT, 040 (2007), arXiv:physics/0703039 [physics.data-an].

[18] T. Junk, Nucl. Instrum. Meth. A 434 (1999) 435. 